\title{
SIM Lite astrometric observatory for detection of Earth-like planets
}

\author{
Xiaopei Pan, Michael Shao and Renaud Goullioud \\ Jet Propulsion Laboratory, California Institute of Technology \\ 4800 Oak Grove Dr, Pasadena, CA 91109, USA \\ email: xiaopei.pan@jpl.nasa.gov, michael.shao@jpl.nasa.gov, renaud.goullioud@jpl.nasa.gov
}

\begin{abstract}
The SIM Lite Astrometric Observatory is an optical astrometry mission for detection of Earth-like planets and investigation of dark matter, galaxy assembly, black holes etc. SIM Lite is a minimum system that performs the full NRC recommenced SIM science programs. This paper summarize the latest status and progresse of the SIM Lite space mission.
\end{abstract}

\section{SIM Lite performance}

The SIM Lite mission is based on development of the SIM PlanetQuest project and has completed a ten-year technology program which was confirmed by multiple external independent reviews. SIM Lite is a cost-effective system, and has passed eight technology gates in component technology, subsystem level testing and system level testing. The SIM Lite instrument includes a $6 \mathrm{~m}$ Michelson Stellar Interferometer (MSI) with a $50 \mathrm{~cm}$ science siderostat aperture at visual bands (450-950 nm). Another $4 \mathrm{~m}$ MSI and a $30 \mathrm{~cm}$ telescope are used for the guide system. The SIM Lite spacecraft will use an Earth-trailing solar orbit with five year mission time, which can be extended to ten years. The global astrometry accuracy of the SIM Lite mission is $4 \mu$ as. The narrow angle astrometry has accuracy of $1 \mu \mathrm{as}$ for a single measurement, and $0.04 \mu \mathrm{as}$ for mission accuracy.

\section{Detecting Earth-analogs around nearby FGK stars}

The SIM Lite mission is an astrometric exoplanet search recommended by the Exoplanet Task Force (AAAC2008), and is free of the limits of astrophysical noise, which is the main limiting factor for radial velocity techniques now. SIM Lite is the only mission which can detect Earth-like planets around nearby FGK stars. Planet surveys by the SIM Lite mission are divided into three categories: the Deep Survey observes 60 stars within 10 pc for Earth analogs; the Broad Survey will search about 2000 stars for planets with mass of a few Earths and greater; and the Young Planet Survey will study about 200 stars of 1-100 Myr ages. Figure 1 compares SIM Lite with other techniques, and demonstrates SIM Lite's capabilities for rocky planet detection.

In particular SIM Lite has the unique capability to search for exoplanets around binary stars. Current explorations for exoplanets are biased to avoid binary stars because the signatures of two stellar objects confuse the tiny signals of planets. Spectroscopic binaries with separation of 11 mas or less will not be resolved by the SIM Lite instrument and can be treated as one star for planet detection. The movements caused by stellar orbital motions can be modeled and easily removed afterwards. In fact wide binary stars (a few arcsecond or more) will use the same sets of reference stars in SIM Lite observation, and can save $80 \%$ of the observation time for the second object. SIM Lite definitely is a powerful tool to discover the diversity of planetary system architectures. 


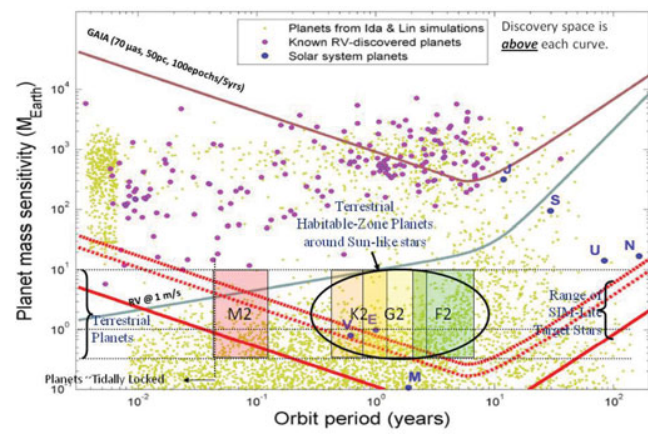

Figure 1. SIM Lite detection of Earth-analogs for nearby FGK stars

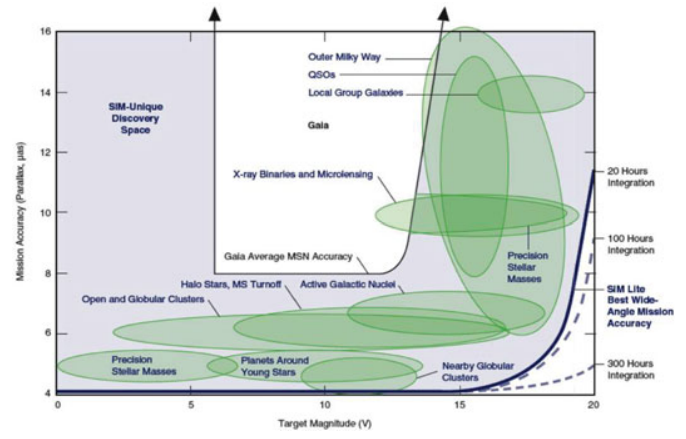

Figure 2. SIM Lite wide-angle discovery space over bright and faint objects

A NASA-directed double-blind, planet-finding capability study (DBS) was conducted in 2008. The study ( Shao et al. 2009 ) confirms the predicted SIM Lite capability for detection of Earth-like planets even in complex planetary systems. It has been demonstrated that SIM Lite can detect multiple planets down to one Earth mass around the nearest 60 FGK stars, and meets the astrometric mission recommendation of the AAAC Exoplanet Task Force.

\section{Precision astrometry over bright and faint objects}

SIM Lite represents an entirely new measurement capability in astronomy and a revolution in astrometry that will exceed all previous measurements by a factor of 100-1,000. SIM Lite has a target-oriented instrument which has flexible integration time for bright targets $(V=-1.5 \mathrm{mag})$ or faint targets $(V=20 \mathrm{mag})$. Also, SIM Lite can easily adjust its observing schedule to respond to Target of Opportunity. Unprecedented high accuracy ( $4 \mu \mathrm{as})$ for wide-angle astrometry by SIM Lite provides the best opportunity to explore dark matter, the dynamics of galaxies, astrometric microlensing, massive black holes, and so on. The unique discovery space of SIM Lite in astrophysics is shown in Figure 2 (see details in Davidson et al. 2009).

\section{Conclusions}

SIM Lite is the only mission concept that is ready to go now, and is capable of finding one-Earth-mass planets in the habitable zone of nearby Sun-like stars. SIM Lite's unique capability for detection of exoplanets in binary star systems provides an excellent opportunity to study architectures and evolution of planets. Three survey categories of SIM Lite provide fundamental statistics of exoplanets for future missions. In addition SIM Lite will map the local dark matter, probe the Milky Way, and investigate supermassive black holes.

\section{References}

Shao, M., Zhai, C., Catanzarite, J., Loredo, T., McArthur, B., \& Benedict, F. 2009, BAAS 41, 268

Davidson, J., Unwin, S., Edberg, S., Danner, R., \& Nemati, B. (eds.) 2009, SIM Lite Astrometric Observatory, JPL Publication 400-1360 